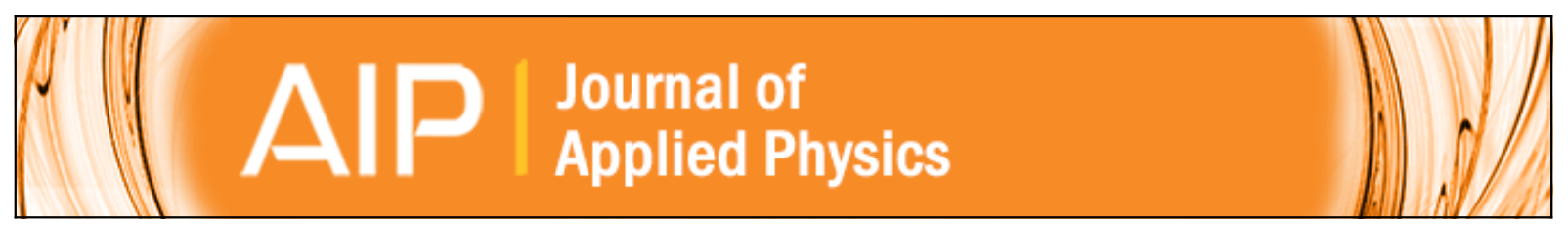

\title{
Seebeck coefficient of one electron
}

Zahid A. K. Durrani

Citation: Journal of Applied Physics 115, 094508 (2014); doi: 10.1063/1.4867775

View online: http://dx.doi.org/10.1063/1.4867775

View Table of Contents: http://scitation.aip.org/content/aip/journal/jap/115/9?ver=pdfcov

Published by the AIP Publishing

$\stackrel{A}{A} \mathbb{P} P$ Re-register for Table of Content Alerts

Create a profile.

Sign up today! 


\title{
Seebeck coefficient of one electron
}

\author{
Zahid A. K. Durrani ${ }^{\text {a) }}$ \\ Department of Electrical and Electronic Engineering, Imperial College London, South Kensington Campus, \\ London SW7 2AZ, United Kingdom
}

(Received 11 December 2013; accepted 24 February 2014; published online 7 March 2014)

\begin{abstract}
The Seebeck coefficient of one electron, driven thermally into a semiconductor single-electron box, is investigated theoretically. With a finite temperature difference $\Delta T$ between the source and charging island, a single electron can charge the island in equilibrium, directly generating a Seebeck effect. Seebeck coefficients for small and finite $\Delta T$ are calculated and a thermally driven Coulomb staircase is predicted. Single-electron Seebeck oscillations occur with increasing $\Delta T$, as one electron at a time charges the box. A method is proposed for experimental verification of these effects. C 2014 AIP Publishing LLC. [http://dx.doi.org/10.1063/1.4867775]
\end{abstract}

The Seebeck coefficient $S$ or thermopower, where a temperature difference $\Delta T$ applied across a material generates an electric potential $\Delta V$ such that $S=\Delta V / \Delta T$, provides a primary measure of the thermoelectric (TE) properties of a material. ${ }^{1-4}$ In recent years, there has been widespread interest in TE effects in semiconductor nanostructures and nanocomposites, ${ }^{3-7}$ for energy generation and scavenging without emission of greenhouse gases. Here, a reduction in the thermal conductivity of the nanostructure, in combination with relatively unaffected electrical conductivity, provides a means to fabricate TE devices with higher efficiency than in bulk materials. ${ }^{7}$ The TE behavior is, nevertheless, created by many particles, with large numbers of electrons involved in charge and heat transfer. However, the possibility of controlling a single or small numbers of electrons in a nanoscale device raises the interesting question: "Can one electron directly generate an observable Seebeck effect?"

Single-electron and quantum dot (QD) devices ${ }^{8-10}$ provide a means to control charge at the one-electron level by using the Coulomb blockade effect. In early theoretical work on Coulomb blockade, Amman et al. ${ }^{11}$ investigated the Seebeck coefficient of a single mesoscopic tunnel junction, in the low temperature difference limit, $S_{0}=\left.\lim _{\Delta T \rightarrow 0} \frac{\Delta V}{\Delta T}\right|_{T}$, where $T$ is the measurement temperature. They found that in an isolated mesoscopic tunnel junction, where the electron dwell time on the junction $\tau_{d}$, the tunneling time $\tau_{t}$, and the external circuit response time $\tau_{r}$ was such that $\tau_{t}<\tau_{d}<\tau_{r}$, the effect of the single-electron charging energy $E_{C}=e^{2} / 2 C$ led to an exponential reduction in $S$ at low $T$, where $k_{B} T$ $\ll E_{c}$. Here, $C$ is the tunnel junction capacitance. At higher temperature, $E_{c}$ was overcome thermally, leading to an increase in $S$ with $T$. However, it was found to be very difficult to experimentally measure single-electron effects using only a single tunnel junction, as these were overcome by charge fluctuations in the external circuit. ${ }^{8}$

In contrast to a single tunnel junction, the Seebeck coefficient $S_{Q D}$ in QDs has been extensively investigated, both theoretically and experimentally. ${ }^{12-16}$ These works also concentrated on the limit $\Delta T \rightarrow 0$. In a QD with total

${ }^{a)}$ Electronic mail: z.durrani@imperial.ac.uk capacitance $C$, coupled by two tunnel junctions to source/ drain electron reservoirs and capacitively to a gate terminal, a Coulomb energy gap $E_{c}=e^{2} / C$ exists at the Fermi energy $E_{F}$ of the reservoirs. With temperature difference $\Delta T$ between the source and drain, application of a gate voltage shifts the Coulomb gap relative to the Fermi energy, leading to sawtooth oscillations in $S$ with gate voltage. The oscillation peak/ valley ratio $\sim e / 2 T C$, where $T$ is the temperature of the "cold" reservoir. Although each sawtooth is associated with one electron added to the QD, in this configuration many electrons are transferred from the "hot" to the "cold" reservoir.

$S_{Q D}$ may be contrasted with $S$ in lightly doped "bulk" semiconductor material, ${ }^{2,17}$ which depends on the conduction or valance band offset (for $n$-type or $p$-type material, respectively) to the Fermi energy. In a bulk semiconductor, ${ }^{17}$ $S$ is given by $S=\mp \frac{k_{B}}{e}\left(\frac{E_{C / F}-E_{F / V}}{k_{B} T}+\left(r+\frac{5}{2}\right)\right)$, where $k_{B}$ is the Boltzmann constant, $E_{F}$ the Fermi energy, $E_{C}$ and $E_{V}$ the conduction and valance band energies, $e$ the electron charge, and $r$ a scattering factor for charge carriers. The negative/ positive signs are taken for electrons/holes. Typically, $r$ is assumed to have a value between -0.5 and +1.5 , with the former value corresponding to a scattering mechanism dominated by phonon scattering and the latter by impurity scattering. For a lightly doped semiconductor, the band offset $E_{C / F}-E_{F / V}$ can form the majority contribution to $S$. In contrast, in a heavily doped semiconductor or metal $S=\frac{\pi^{2}}{3} \frac{k_{B}^{2}}{e}$ $T\left(\frac{\partial \ln \sigma(E)}{\partial E}\right)_{E=E_{F}}$, where $\sigma$ is the electrical conductivity. ${ }^{17}$ This equation does not depend on the band-offset and $S$ has a far smaller value. If we now compare a semiconductor QD to bulk semiconductor material, the Coulomb gap can be regarded as analogous to the band-offset in bulk material and for both situations, $S$ provides a measure of the potential energy carried by many electrons, and of fundamental charge and heat transfer properties for the system. Even with a metallic "island," the presence of single-electron charging effects opens up a Coulomb gap at the Fermi energy and creates a situation analogous to the band-offset in bulk semiconductors, increasing the value of $S$.

This paper investigates theoretically the Seebeck effect in a semiconductor single-electron box, ${ }^{18}$ consisting of an 
island coupled by a tunnel junction to a source reservoir, and by a storage capacitor to a drain reservoir. In this device, electrons can be added one by one to a semiconductor island at equilibrium, allowing investigation of the Seebeck effect created directly by one, or a few electrons. Furthermore, the use of a semiconductor single-electron box allows us to investigate the variation of the Seebeck effect with the bandoffset and results in an enhancement of the Seebeck coefficient. In contrast to earlier works in the limit $\Delta T \rightarrow 0$, a finite temperature difference $\Delta T$ is applied across the tunnel junction to thermally overcome Coulomb blockade and drive electrons into the box. This leads directly to an island voltage and a corresponding "single-electron Seebeck effect." The Seebeck coefficients $S_{O}$ and $S$ for both small and finite $\Delta T$, and the differential Seebeck coefficient $S_{\text {diff }}=\frac{d(\Delta V)}{d(\Delta T)}$ are investigated as a function of $\Delta T$, and island temperature $T$. If $T$ is small such that $k_{B} T<E_{c}$, the average electron number $\langle n\rangle$ increases in discrete steps as $\Delta T$ increases, creating a thermally driven Coulomb staircase. Corresponding singleelectron Seebeck oscillations are observed in $S$ and $S_{\text {diff, with }}$ larger peak magnitude for $S_{\text {diff. }}$. As the band-offset $\zeta=E_{C}$ $-E_{F}$ is increased, the peak magnitude of $S_{\text {diff }}$ is also seen to increase, implying that larger values of $S_{\text {diff }}$ are possible in a semiconductor single-electron box by controlling $\zeta$. Finally, unlike the oscillations of $S_{Q D}$ in a quantum dot, where many electrons transfer between hot and cold reservoirs and the Coulomb gap is controlled by application of a gate voltage, each oscillation of $S$ and $S_{\text {diff }}$ is generated directly by the addition of one extra electron to the island.

Figure 1(a) shows a single-electron box, formed by a tunnel capacitor $C_{1}$ in series with a storage capacitor $C_{b}$, with an intermediate island region. Figure 1(b) shows schematically the energy band diagram across the device. Here, the right hand side potential barrier corresponds to $C_{b}$ and is assumed to be wide enough to inhibit electron tunnelling. The single-electron charging energy for this device is $E_{c}=e^{2} / 2 C$, where $C=C_{1}+C_{b}$. If $T$ is small such that $E_{c}$ $\gg k_{B} T$, then $E_{c}$ prevents electron tunneling on to the island across $C_{1}$. However, if a temperature difference $\Delta T_{S D}=T_{S}$ $-T_{D}$ is applied across the device, with the source as the "hot" side at temperature $T_{S}$ and the drain as the "cold" side at temperature $T_{D}$, then it becomes possible for thermally
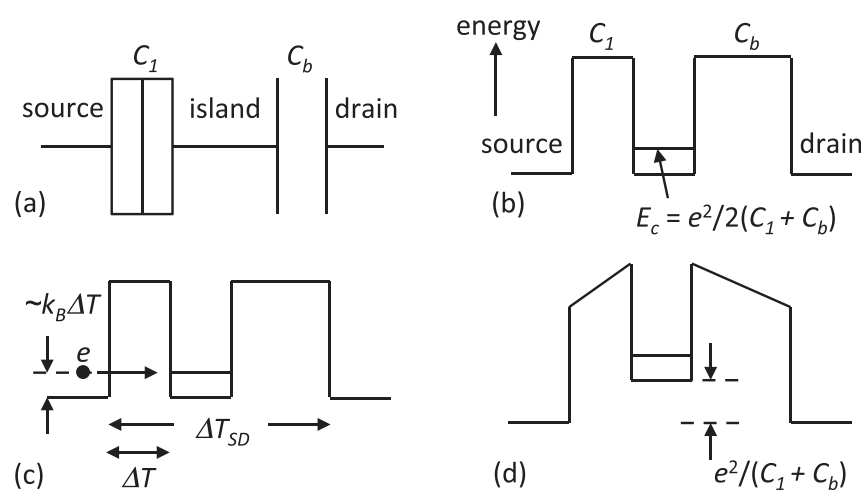

FIG. 1. (a) Single-electron box. (b) Energy diagram across the singleelectron box. The single-electron charging energy is $E_{c}$. (c) Temperature difference $\Delta T$ thermally overcomes Coulomb blockade and drives an electron on to the island. (d) Energy diagram with one extra electron on the box. activated electrons to tunnel on to the island (Fig. 1(c)). For temperature difference $\Delta T=T_{S}-T$ across $C_{1}$, this occurs when $k_{B} \Delta T \sim E_{c}=e^{2} / 2 C$. The average island electron number then increases by one, and the island potential relative to the source becomes $\Delta V=-e / C$ (Fig. 1(d)), inhibiting tunnelling of a second electron. It is then possible to associate a Seebeck coefficient with one electron on the island, $S_{l}=\Delta V / \Delta T \sim k_{B} \Delta V / E_{c} \sim-2 k_{B} / e$. Here, as electrons are transferred, $S_{l}$ is defined to be negative. This quantity depends on fundamental constants only and is independent of material or device parameters.

We now calculate $S, S_{0}$, and $S_{\text {diff }}$ in an $n$-type semiconductor single-electron box, by extending the approach used by Amman et al. ${ }^{11}$ to calculate the Seebeck coefficient of a single tunnel junction with metal contacts. Here, $S$ and $S_{\text {diff }}$ are more relevant experimentally, as there is always a finite $\Delta T$ in a measurement. The Seebeck coefficients are defined as

$$
S=\left.\frac{\langle V\rangle}{\Delta T}\right|_{T}, S_{0}=\left.\lim _{\Delta T \rightarrow 0} \frac{\langle V\rangle}{\Delta T}\right|_{T}, S_{\text {diff }}=\left.\frac{d\langle V\rangle}{d(\Delta T)}\right|_{T} .
$$

$\langle V\rangle$ is the average voltage of the island relative to the source, $\Delta T=T_{S}-T, T_{S}$ the source temperature and $T$ the island temperature. Regarding $S_{0}$, while this is defined for $\Delta T \rightarrow 0$, in practice a finite $\Delta T$ must be present to drive the single-electron box, and similar to the approach of Amman et al. for a single tunnel junction, ${ }^{11}$ in the following we calculate $S_{O}$ for small $\Delta T$ $\ll T$. With the condition $\Delta T>0,\langle n\rangle$ and $\langle V\rangle$ are given by

$$
\begin{gathered}
\langle n\rangle=\sum_{n=0}^{\infty} n \rho_{e}(n), \\
\langle V\rangle=\frac{\langle n\rangle e}{C}=\frac{e}{C} \sum_{n=0}^{\infty} n \rho_{e}(n) .
\end{gathered}
$$

$\rho_{e}(n)$ is the steady state probability for $n$ electrons on the island and in our case, $n \geq 0$, as $\Delta T>0$ implies that electrons are only added to the island. $\rho_{e}(n)$ is given by the steady state solution to the master equation

$$
\begin{aligned}
\frac{\partial \rho(n, t)}{\partial t}= & r(n+1) \rho(n+1, t)+l(n-1) \rho(n-1, t) \\
& -(r(n)+l(n)) \rho(n, t)=0
\end{aligned}
$$

$r(n)$ and $l(n)$ are the electron tunneling rates across the tunnel junction from the right (i.e., the island), and from the left (i.e., the source), respectively. $l(n)>0$ leads to electrons tunneling on to the island, and $r(n)>0$ leads to electrons leaving the island. Equation (4) comprises the probability rates for the transition from $n+1$ or $n-1$ to $n$ electrons on the island, minus the rates with $n$ electrons on the island. Assuming a constant, normalised density of states $D$ on both the island and the source, these tunneling rates are given by

$$
r(n)=\frac{D^{2}}{e^{2} R} \int_{\left(n e^{2} / C\right)+\zeta}^{\infty} f^{T}\left(E-\frac{n e^{2}}{C}\right)\left(1-f^{T+\Delta T}\left(E-\frac{e^{2}}{2 C}\right)\right) d E,
$$




$$
l(n)=\frac{D^{2}}{e^{2} R} \int_{\left(n e^{2} / C\right)+\zeta}^{\infty} f^{T+\Delta T}(E)\left(1-f^{T}\left(E-\frac{n e^{2}}{C}-\frac{e^{2}}{2 C}\right)\right) d E .
$$

Here, $R$ is the tunnel junction resistance, $\zeta=E_{C}-E_{F}$ is the conduction band offset, and $f^{T}(E)=1 /\left(1+\exp \left(E / k_{B} T\right)\right)$ is the Fermi-Dirac distribution function, at temperature $T$ and with energy $E$ defined relative to the Fermi energy $E_{F}$. The term $n e^{2} / C$ corresponds to the increase in the energy of the island with $n$ extra electrons on the island, and the term $e^{2} / 2 C$ is the single-electron charging energy. Furthermore, the integrals are evaluated from $n e^{2} / C+\zeta$ to infinity, as this is the minimum energy of electrons on the island (relative to $E_{F}$ ) with $n$ electron on the island. The minimum energy for electrons on the source is $\zeta$ and the situation for one electron on the island is shown in Fig. 1(d). While electrons can exist on the source at lower energies than $n e^{2} / C+\zeta$, if $n>0$ and as long as $E>\zeta$, these electrons cannot tunnel onto the island as their energies overlap with either the band gap or the Coulomb gap in the island.

We now use the Boltzmann approximation to solve Eqs. (5) and (6). The integrals may then be solved directly for $\zeta$ $\gg k_{B} T$, using $f^{T}(E) \approx \exp \left(-E / k_{B} T\right)$, and allowing evaluation of the following explicit results:

$$
\begin{aligned}
r(n)= & \frac{D^{2}}{e^{2} R}\left(k_{B} T \exp \left(-\frac{\zeta}{k_{B} T}\right)-\frac{k_{B} T(T+\Delta T)}{2 T+\Delta T}\right. \\
& \left.\times \exp \left(-\frac{\zeta(2 T+\Delta T)+\frac{e^{2}}{C}\left(n-\frac{1}{2}\right) T}{k_{B} T(T+\Delta T)}\right)\right),
\end{aligned}
$$

Using Eqs. (7) and (8), in a manner similar to the solution of Eq. (4) given by Amman et al. ${ }^{11,19}$ for the normalisation condition $\sum_{0}^{\infty} \rho_{e}(n)=1$ the steady state probability $\rho_{e}(n)$ is given by

$$
\begin{gathered}
\rho_{e}(0)=\frac{\left(\prod_{j=1}^{\infty} r(j)\right)}{\prod_{m=1}^{\infty} r(m)+\sum_{k=1}^{\infty}\left(\prod_{j=0}^{k-1} l(j)\right)\left(\prod_{j=k+1}^{\infty} r(j)\right)}, \quad \text { (9a) } \\
\rho_{e}(n)=\frac{\left(\prod_{j=0}^{n-1} l(j)\right)\left(\prod_{j=n+1}^{\infty} r(j)\right)}{\prod_{m=1}^{\infty} r(m)+\sum_{k=1}^{\infty}\left(\prod_{j=0}^{k-1} l(j)\right)\left(\prod_{j=k+1}^{\infty} r(j)\right)} \quad \text { For } n \geq 1 .
\end{gathered}
$$

Equations (9a) and (9b) may be evaluated numerically, using Eqs. (7) and (8). Equations (1)-(3) can then be used to find $\langle n\rangle$ and hence $S, S_{0}$, and $S_{\text {diff. }}$.

Figure 2(a) shows $\langle n\rangle$ (Eq. (2)) and $S_{0} e / k_{B}$ vs. dimensionless island temperature $T k_{B} / E_{c}$, with small $\Delta T$. Here, $S_{0}$ and $T$ are plotted in dimensionless forms relative to $e / k_{B}$ and $k_{B} / E_{c}$, respectively. Curves are plotted for a constant ratio $\alpha=\Delta T / T$, for $\alpha=0.1,0.05$, and 0.01. For small $T \ll E_{c} / k_{B}$, the island is in Coulomb blockade, $\langle n\rangle \sim 0$ and a Coulomb gap is observed with $T$. $\langle n\rangle$ tends exponentially to zero as $T$ tends to zero (Fig. 2, inset) and tends to increase linearly with $T$ when $k_{B} T>E_{c}$. For intermediate values of $T$, Coulomb blockade is overcome at lower $T$ for larger values of $\alpha$. In Fig. 2(a), Coulomb blockade is overcome thermally by increasing island temperature. $\Delta T$ corresponds only to a small temperature difference between the source and the island, and cannot overcome Coulomb blockade by itself.
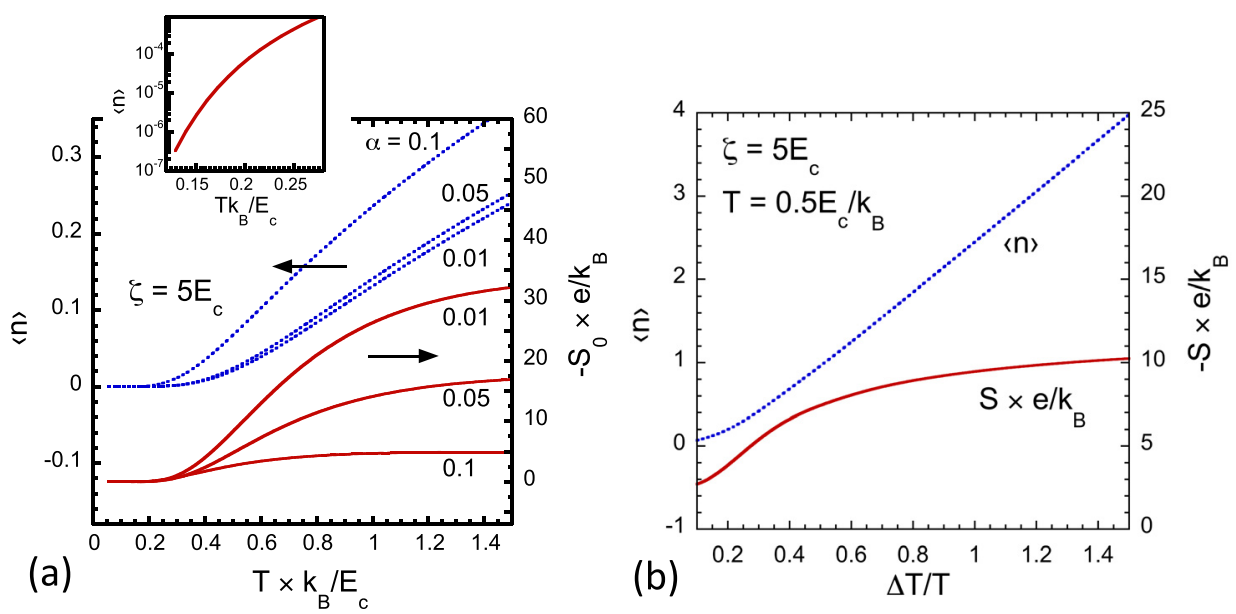

FIG. 2. (a) Average electron number $\langle n\rangle$ (dashed lines), and dimensionless Seebeck coefficient $S_{0} e / k_{B}$ in the low $\Delta T$ limit (solid lines) vs. dimensionless island temperature $T k_{B} / E_{c}$. Curves shown for three values of $\alpha=\Delta T / T$. Inset: $\langle n\rangle$ reduces exponentially for low $T$. (b) $\langle n\rangle$ (dashed line) and dimensionless Seebeck coefficient $S e / k_{B}$ for finite $\Delta T$ (solid line) vs. $\Delta T / T . T$ is high enough to allow Coulomb blockade to be easily overcome.

(b) 

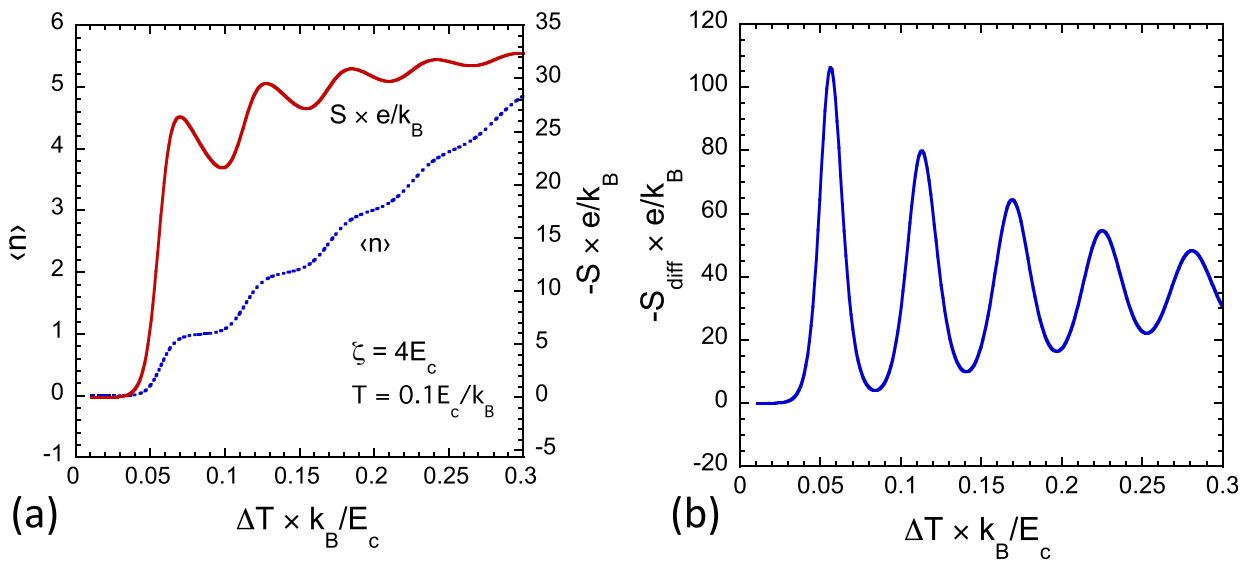

FIG. 3. (a) Thermally driven Coulomb staircase in $\langle n\rangle$ and single-electron Seebeck oscillations in $S e / k_{B}$ vs. $\Delta T k_{B} / E_{c}$. The single-electron box is in the Coulomb blockade regime. (b) Single-electron Seebeck oscillations in $S_{\text {diff }}$ vs. $\Delta T k_{B} / E_{c}$, corresponding to the conditions in (a).
Therefore, $\langle n\rangle$ does not show any quantisation, as $E_{c}$ is always thermally overcome by $T$. In practice, the continuous variation of $\langle n\rangle$ simply represents a displacement in the electron density on the island with respect to the background ion density. ${ }^{18}$ In a manner similar to $\langle n\rangle, S_{O}$ shows a Coulomb gap at low $T$. However, as $T$ increases, $S_{0}$ saturates. This is a consequence of constant $\alpha$ in each curve, implying that $\Delta T$ increases linearly with $T$. As $\langle n\rangle$ and $\langle V\rangle$ (Eq. (3)), both tend to increase linearly at higher $T$, the ratio $\langle V\rangle / \Delta T$ tends to saturate, with higher magnitudes for larger values of $\alpha$.

Figure 2(b) shows $\langle n\rangle$ and $S e / k_{B}$ vs. $\Delta T / T$, for constant $T=E_{c} / 2 k_{B}$. Here, $T$ is large enough to overcome Coulomb blockade, and we plot $S=\langle V\rangle / \Delta T$ as $\Delta T$ can be large and no longer tends to zero. Larger values of $\Delta T$, from $0.1 \mathrm{~T}$ to $1.5 \mathrm{~T}$, lead to greater values of $\langle n\rangle$ on the island, though as before, quantisation is not observed. Again, $\mathrm{Se} / k_{B}$ saturates at higher temperatures, though this is driven by $\Delta T$ and not $T$.

We now use $\Delta T$ to drive a single-electron box within the Coulomb blockade regime, where $k_{B} T<E_{c}$. Figure 3(a) shows $\langle n\rangle$ and $S e / k_{B}$ vs. $\Delta T k_{B} / E_{c}$, for constant $T=0.1 E_{c} / k_{B}$, and Fig. 3(b) shows the corresponding curve for $S_{\text {diff }}(\mathrm{Eq}$. (1)). As $T$ is small enough for the island to be in Coulomb blockade, $\Delta T$ is may be regarded as equivalent to a thermal voltage, as discussed qualitatively for Fig. 1. $\langle n\rangle$ shows a thermally driven Coulomb staircase, and increases in discrete values. The steps are thermally washed out at higher $\Delta T$, as $k_{B} \Delta T$ begins to approach $E_{c}$. The curve for $S$ shows the Seebeck coefficient for one to a few electrons. In contrast to $\langle n\rangle$, single-electron Seebeck oscillations are observed in $S$, with larger peak-valley ratio for small $\Delta T$. In Fig. 3(b), $S_{\text {diff }}$ shows even stronger oscillations with $\Delta T$. Each oscillation

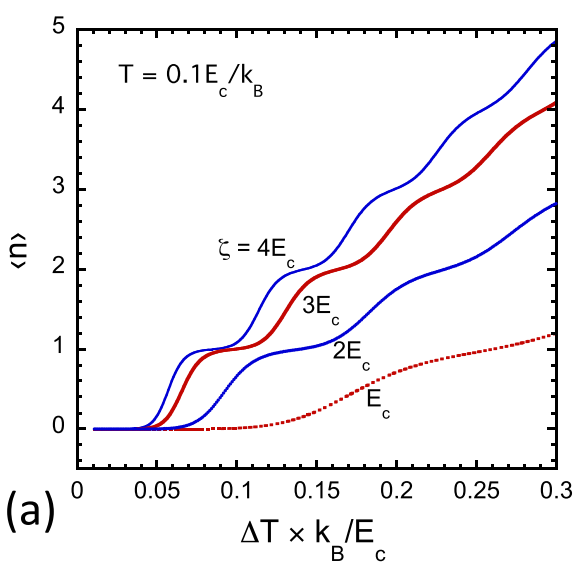

(b)
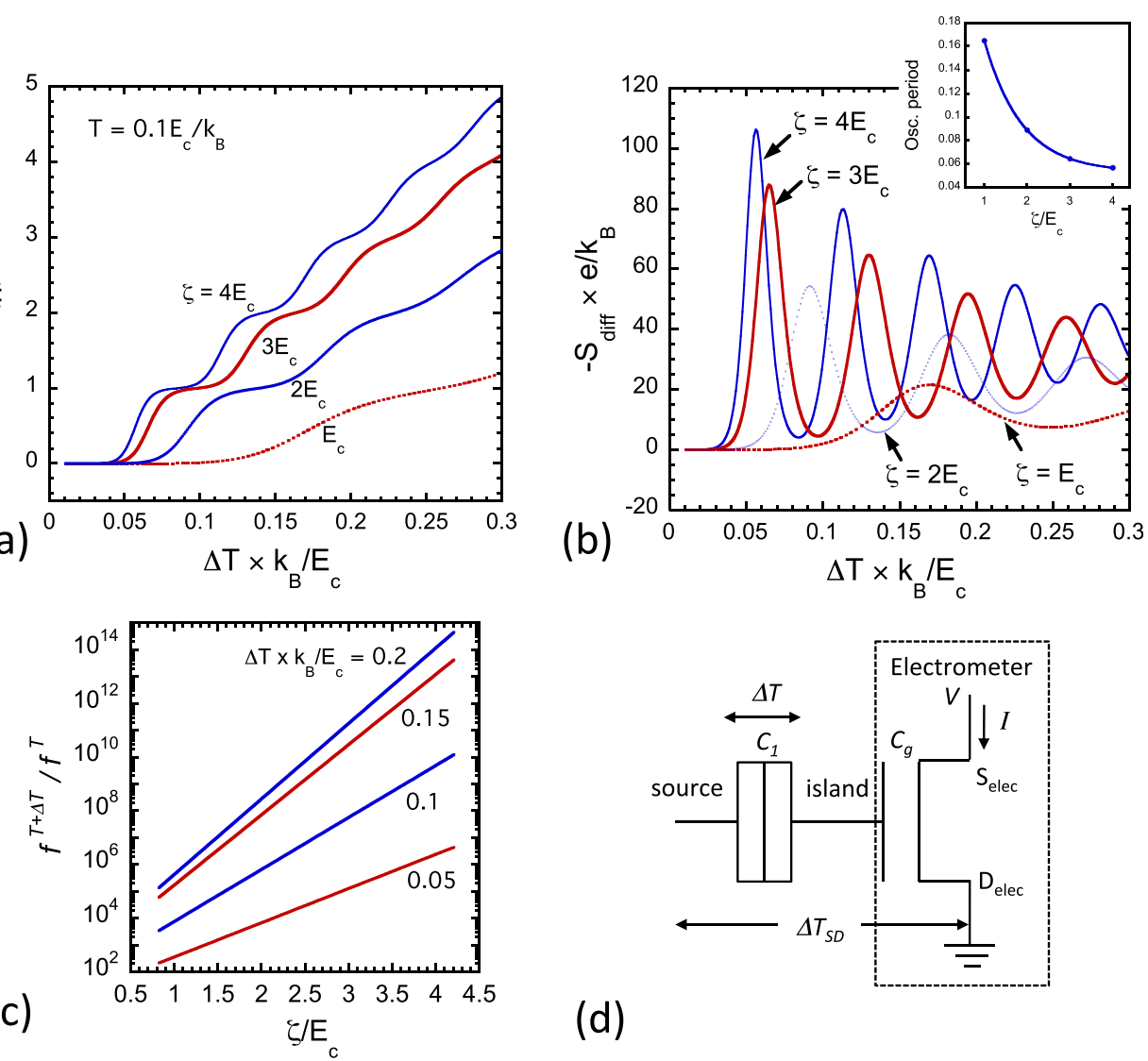

FIG. 4. (a) Thermally driven Coulomb staircase in $\langle n\rangle$ vs. $\Delta T k_{B} / E_{c}$, for various band offset $\zeta$. (b) Single-electron Seebeck oscillations in $S_{\text {diff }}$ vs. $\Delta T k_{B} / E_{c}$, for various band offset $\zeta$. Inset: Oscillation period $\Delta T_{o s c} k_{B} / E_{c}$ vs. $\zeta / E_{c}$. (c) Ratio of Boltzmann distributions for source and island at energy $E=\zeta+E_{c}$, as a function of $\zeta / E_{c}$, with $\Delta T \times k_{B} / E_{c}$ $=0.05-0.2$. (d) Proposed circuit for experimental measurement. 
corresponds to the addition of one electron to the island. The peak magnitude of $S_{\text {diff }}$ may be much greater than $S$. The largest difference is for the first peak, which is $\sim 4$ times

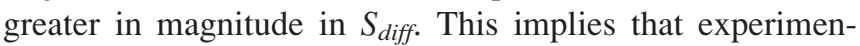
tally, if $\Delta T$ cannot be reduced to zero, a much larger Seebeck coefficient may be observed for small, additional variation in $\Delta T$.

Figure 4 shows, for varying band offset $\zeta$, thermally driven Coulomb staircases in $\langle n\rangle$ (Fig. 4(a)), and oscillations in $S_{\text {diff }}$ vs. $\Delta T k_{B} / E_{c}$ (Fig. 4(b)). Figure 4(b) inset shows the oscillation period $\Delta T_{\text {osc }}$ of $S_{\text {diff }}$ as a function of $\zeta$. For larger $\zeta$, both the staircase and oscillations are stronger and may be observed at lower $\Delta T$. This is caused by the behavior of the exponential tail of the Boltzmann distribution on the source, compared to that on the island. To illustrate this, Fig. 4(c) shows the ratio of the Boltzmann distributions $f^{T+\Delta T}(E) / f^{T}(E)$ at energy $E=\zeta+E_{c}$, as a function of $\zeta / E_{c}$, or an island with $E_{c}=0.8 \mathrm{meV}, \quad T=1 \mathrm{~K}, \quad$ and $\Delta T \times k_{B} / E_{c}=0.05-0.2$. Here, the ratio considers a state on the source with energy equal to the single-electron energy of the island. When $\zeta / E_{c}$ is increased, the ratio increases exponentially, implying that it is much easier to thermally occupy states in the source, relative to the island, when $\zeta$ is larger. This causes a decrease in the oscillation period and an increase in magnitude for $S_{\text {diff }}$ as $\zeta / E_{c}$ increases (Fig. 4(b)).

Recognising that the electrical properties of the singleelectron box, ${ }^{18}$ and single-electron memory cells based on this ${ }^{20-22}$ have been extensively investigated, it should be possible to experimentally verify the theoretical predictions of this paper. In experimental work on the single-electron box/memory, a single-electron transistor or field-effect transistor is used as an electrometer to sense the island voltage through the transistor drain-source current $I$ (Fig. 4(d)). The storage capacitor $C_{b}$ is then formed by the transistor gate capacitor $C_{g}$. It is proposed that for Seebeck measurements, a temperature difference $\Delta T_{S D}$ applied between the tunnel junction source and the electrometer drain $\left(D_{\text {elec }}\right)$ is used to create $\Delta T$ and drive electrons on the island. $\Delta T_{S D}$ may be generated using a resistive heater (e.g., similar to QD work, (Ref. 14)). Oscillations should then be observed in $I$ vs. $\Delta T$, corresponding to single-electron Seebeck oscillations in $S_{\text {diff }}$. Finally, we note that in experimental work on semiconductor single-electron devices, ${ }^{20-22}$ it is common for the source and drain electrodes to be heavily doped, to allow electrical contact at cryogenic temperatures. Calculation with a heavily doped source would require use of the full Fermi-Dirac distribution. However, if $E_{c} \gg k_{B} T_{S}$ then only electrons from the distribution tail are likely to tunnel onto the island and the use of a Boltzmann approximation remains valid.

In summary, a Seebeck effect can be generated directly by single electrons, driven thermally, one by one, into a single-electron box. With temperature difference $\Delta T$ across the tunnel junction of the box, one electron can charge the island in equilibrium and directly generate a Seebeck effect. Seebeck coefficients for small and finite $\Delta T$, and the differential Seebeck coefficient, are investigated. At small island temperature, a thermally driven Coulomb staircase is observed for island electron number. Single-electron Seebeck oscillations occur with $\Delta T$, as the box charges one electron at a time.

The author would like to thank M. E. Jones and W. T. Pike for valuable discussions in the preparation of this work. The research leading to these results has received funding from the European Union's Seventh Framework Programme FP7/2007-2013 under Grant Agreement No. 318804.

\footnotetext{
${ }^{1}$ A. Majumdar, Science 303, 777-778 (2004).

${ }^{2}$ G. S. Nolas, J. Sharp, and H. J. Goldsmid, Thermoelectrics: Basic Principles and New Material Developments (Springer-Verlag, Berlin, Heidelberg, 2001).

${ }^{3}$ F. DiSalvo, Science 285, 703-706 (1999).

${ }^{4}$ M.-S. Dresselhaus, G. Chen, M. Y. Tang, R. G. Yang, H. Lee, D. Z. Wang, Z. F. Ren, J.-P. Fleurial, and P. Gogna, Adv. Mater. 19, 1043 (2007).

${ }^{5}$ A. I. Hochbaum, R. Chen, R. D. Delgado, W. Liang, E. C. Garnett, M. Najarian, A. Majumdar, and P. Yang, Nature 451, 163 (2008).

${ }^{6}$ E. Krali and Z. A. K. Durrani, Appl. Phys. Lett. 102, 143102 (2013).

${ }^{7}$ T. C. Harman, P. J. Taylor, M. P. Walsh, and B. E. LaForge, Science 297, 2229 (2002).

${ }^{8}$ K. K. Likharev, Proc. IEEE 87, 606 (1999).

${ }^{9}$ Z. A. K. Durrani, Single-Electron Devices and Circuits in Silicon (Imperial College Press, London, 2010).

${ }^{10}$ L. P. Kowenhoven, C. M. Marcus, P. L. McEuen, S. Tarucha, R. M. Westervelt, and N. S. Wingreen, Mesoscopic Electron Transport, Electron Transport in Quantum Dots, edited by L. L. Sohn, L. P. Kowenhoven, and G. Schön (Kluwer, Dordrecht, 1997).

${ }^{11}$ M. Amman, E. Ben-Jacob, and J. Cohn, Z. Phys. B: Condens. Matter 85, 405 (1991).

${ }^{12}$ C. W. J. Beenakker and A. A. M. Staring, Phys. Rev. B 46, 9667 (1992).

${ }^{13}$ X. Chen, H. Buhmann, and L. W. Molenkamp, Phys. Rev. B 61, 16801 (2000).

${ }^{14}$ L. W. Molenkamp, A. A. M. Staring, B. W. Alphenaar, H. van Houten, and C. W. Beenakker, Semicond. Sci. Technol. 9, 903 (1994).

${ }^{15}$ A. S. Dzurak, C. G. Smith, M. Pepper, D. A. Ritchie, J. E. F. Frost, G. A. C. Jones, and D. G. Hasko, Solid State Commun. 87, 1145 (1993).

${ }^{16}$ R. Scheibner, H. Buhmann, D. Reuter, M. N. Kiselev, and L. W. Molenkamp, Phys. Rev. Lett. 95, 176602 (2005).

${ }^{17}$ L. Weber and E. Gmelin, Appl. Phys. A: Mater. Sci. Process. 53, 136 (1991).

${ }^{18}$ P. Lafarge, H. Pothier, E. R. Williams, D. Esteve, C. Urbina, and M. H. Devoret, Z. Phys. B: Condens. Matter 85, 327 (1991).

${ }^{19}$ M. Amman, R. Wilkins, E. Ben-Jacob, P. D. Maker, and R. C. Jaklevic, Phys. Rev. B 43, 1146 (1991).

${ }^{20}$ K. Nakazato, R. J. Blaikie, and H. Ahmed, J. Appl. Phys. 75, 5123 (1994).

${ }^{21}$ Z. A. K. Durrani, A. C. Irvine, and H. Ahmed, IEEE Trans. Electron Devices 47, 2334 (2000).

${ }^{22}$ Y. Takahashi, Y. Ono, A. Fujiwara, and H. Inokawa, J. Phys.: Condens.
} Matter 14, R995 (2002). 